\title{
Comparison and Selection of Sewage Outlet of a Refining and Chemical Project Based on Mike21 Model
}

\author{
Li Yajuan ${ }^{1}$, Zhu Yuxin ${ }^{1}$, and Mao Tianyu ${ }^{1 *}$ \\ ${ }^{1}$ Laboratory of Waterway Environmental Protection Technology, Tianjin Water Transport Engineering Science Research Institute, \\ Tianjin 300456, China
}

\begin{abstract}
The Mike21 model is used to conduct a numerical simulation study on the setting plan of the warm water outlet of a refining and chemical project. After determining the environmental conditions, formulate key constraints. Through the parameter setting of the model, a hydrodynamic model is established, and numerical simulation of temperature and drainage under several combinations of different schemes is carried out. According to the prediction results of the model, the results under different emission conditions in winter and summer and under different environmental conditions can be compared and analyzed, combined with the requirements of environmental conditions, and optimized, thereby obtaining relatively optimized site selection conditions. Through the process of comparison and selection, the implementation method and application process of the Mike21 model for numerical simulation of warm drainage are proposed, and the application focus of the selection of the location of warm drainage outlets is summarized.
\end{abstract}

\section{INTRODUCTION}

A refining and chemical project is located in the Caofeidian Sea Area of Tangshan. It is planned to use a seawater through-flow cooling system to cool the coolers of the refining and chemical plants, and the seawater after heat exchange is directly discharged back to the sea. The project requires 201.2 million $\mathrm{m} 3 / \mathrm{h}$ of seawater for cooling in summer, and the temperature rise of the discharged seawater after cooling is $10^{\circ} \mathrm{C}$; in winter, the required seawater volume is 1352.6 million $\mathrm{m} 3 / \mathrm{h}$, and the temperature of the discharged seawater rises by $14^{\circ} \mathrm{C}$ after cooling.

There are clauses in the "Sea Water Quality Standards" that the seawater temperature rise caused by man-made in the four types of sea areas should not exceed $4{ }^{\circ} \mathrm{C}$ at the time. Second-class sea areas have more stringent requirements. In order to meet the requirements of the standard, the location of the discharge outlet must be optimized. Through the mathematical model method $^{[1]}$, the tidal current characteristics of the sea area around Caofeidian are analyzed, including the sea area south of Caofeidian Head, Nachao River and Sangangchi. The analysis can be used for The area and location of the temperature discharge outlets of this project; predict the envelope range of the isotherm when reaching equilibrium under various temperature discharge flows and typical tidal level processes; The analysis was carried out simultaneously in conjunction with the surrounding existing warm water vents. Under the condition of discharge, after superimposing the influence of temperature envelope range, the influence range of discharge warm water was analyzed; further compare the model prediction results of different temperature outlet setting schemes, and select the least impact scheme, as Provide the basis for the environmental impact assessment and engineering design of the drainage project of the project.

The Mike21 model designed the entire process of hydrodynamic numerical simulation into a software package, with good pre- and post-processing tools, lowering the technical threshold, and making it highly versatile $^{[2]}$.

\subsection{STUDY AREA}

The Caofeidian Petrochemical Industrial Base is one of the key new petrochemical industrial bases for development. It has been officially included in the "Beijing-Tianjin-Hebei Coordinated Development Plan Outline" and the "Beijing-Tianjin-Hebei 13th Five-Year Plan". As a major project of the Caofeidian Petrochemical Industrial Base, the construction of a certain refining and chemical project discussed this time is conducive to the formation of the industrial agglomeration advantage of the Caofeidian Petrochemical Industrial Base and the construction and development of the petrochemical industrial base. The proposed temperature discharge outlet of this project is located in the sea area near the Caofeidian Petrochemical Industrial Base, and the four types of seawater quality standards are implemented ${ }^{[3]}$.

\footnotetext{
* Corresponding author: maotianyu@foxmail.com
} 


\subsection{ANALYSIS OF RESTRICTIONS}

According to the layout of the Caofeidian port area, Shougang Jingtang Company and China Resources Power Plant are respectively arranged on the west side of the petrochemical industry base, and corresponding water intakes are arranged in a port basin. Therefore, in order to avoid the influence of warm water discharge on the temperature of the water body of the port basin. The warm discharge port cannot be installed inside a harbor basin and needs to be as far away as possible from a harbor basin to ensure that the warm water discharge will not have a significant impact on the temperature of the water in a harbor basin.

On the south side of the petrochemical industry base and in the Diantou area, super-large deep-water piers, deep-water piers, sub-deep-water piers, and mediumdeep-water piers will be arranged. Except for the current pier shore section, this area can be used as a proposed area for warm drainage. The possible Wenpaikou sites are all located in the "Caofeidian Port Shipping Area 26" in Hebei Province's Marine Functional Zoning. The sea water quality standards implemented in the surrounding area are four types of sea water quality standards.

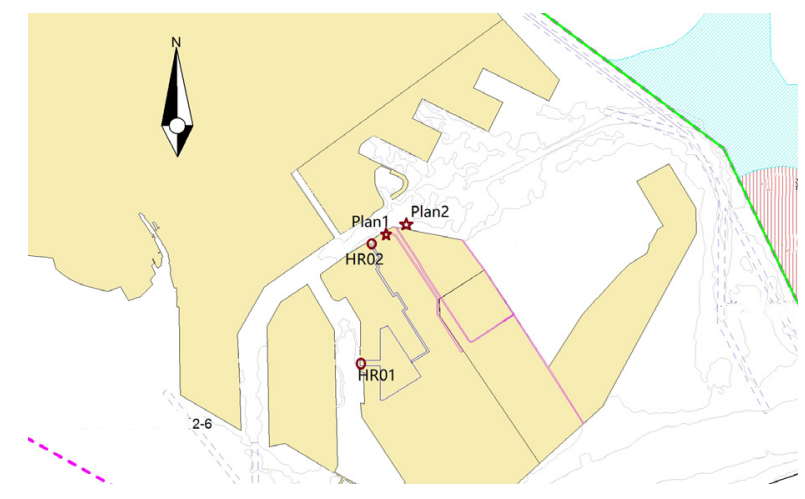

Fig 1 The surrounding conditions of the proposed sewage outfall plan (HR-China Resources Power Plant, Plan-this plan)

\subsection{MODEL RELATED PARAMETERS}

According to the engineering design data, under the condition that the heat exchange area and heat transfer intensity remain unchanged, the sea water pumping station is equipped with 12 pumps, and the summer sea water circulation pump is equipped with 2 pumps. The sea water circulation volume is $201,200 \mathrm{~m} 3 / \mathrm{h}$ (total $55.889 \mathrm{~m} 3 / \mathrm{s}$ ), the seawater temperature rises by $10^{\circ} \mathrm{C}$; in winter, the seawater circulation pump has 6 on and 6 standby, the seawater circulation volume is 1352.6 million $\mathrm{m} 3 / \mathrm{h}$ (total $37.5722 \mathrm{~m} 3 / \mathrm{s}$ ), and the temperature rise is $14^{\circ} \mathrm{C}$.

The tides in the Caofeidian sea area are strong, and the tides are more prominently affected by the topography. There is an obvious difference in the flow velocity between the deep-water area and the shallow tidal current. In the deep-water area, the tidal current is basically parallel to the contour of the sea area. Main [4], the maximum flow velocity is about $1.20 \mathrm{~m} / \mathrm{s}$; the flow velocity in Caofeidian Second Port Pool is relatively weak. From the simulation calculation of spring tide and neap tide, the flow velocity during the neap tide period is slightly lower than that in the spring tide period, especially the low tide performance is more prominent.

\section{MATERIALS AND METHODS}

\subsection{MODEL BUILDING PROCESS}

In order to ensure that the local flow field calculation conforms to the overall physical characteristics of the tidal current field, a double-layer nesting method is adopted for calculation. The models are the Bohai Sea area and the area near the project. The open boundary of the tidal current calculation model is controlled by tide level, and the boundary tide level is obtained by using the harmonic constants of the tidal components at the boundary.

$$
\eta(t)=\sum_{i=1}^{n} H_{i} F_{i} \cos \left[\sigma_{i} t+\left(V_{0}+u\right)_{l}-g_{i}\right]
$$

$(\mathrm{N}=8$ ), (where $\mathrm{F}$ is the correction factor of the tidal amplitude, is the angular velocity of the tidal component, $\mathrm{Vo}+\mathrm{u}$ is the phase angle of the hypothetical celestial body at zero universal time on the day of the observation period. $\mathrm{H}$ and $\mathrm{K}$ are the tidal component Harmonic constant, $\mathrm{H}$ is the tidal height of each sub-tide, $\mathrm{g}$ is the delay angle of each sub-tide. I is each sub-tide, this calculation takes M2, S2, N2, K2, K1, O1, P1, Q1, a total of 8 components). After the Bohai Sea tidal current calculation, the tidal level boundary conditions in the tidal current field calculation of the small area are provided by the calculation results of the upper layer model; the calculation uses a square grid with a grid space step of $120 \mathrm{~m}$. The area near the project is encrypted and the network is encrypted. The grid step length is 40 meters.

The hydrological data adopts the measured data in 2018, among which the current velocity and flow direction data are the measured spring tide data from April 18th to 19th, 2018, and the measured small tide data from May 9th to 10th, 2018. There are 6 tidal current stations; the tide level adopts the synchronous tide station data, A total of 2 tide level stations are set up. The water depth uses the latest sea chart water depth data, and the shoreline comes from satellite image data in December 2017. Taking into account that the overall project has begun operation and production in the normal operation stage, and the related supporting wharf engineering and channel engineering have also been completed and put into production, the water depth in local areas such as docks and waterways is predicted by using the planned and designed water depth. 


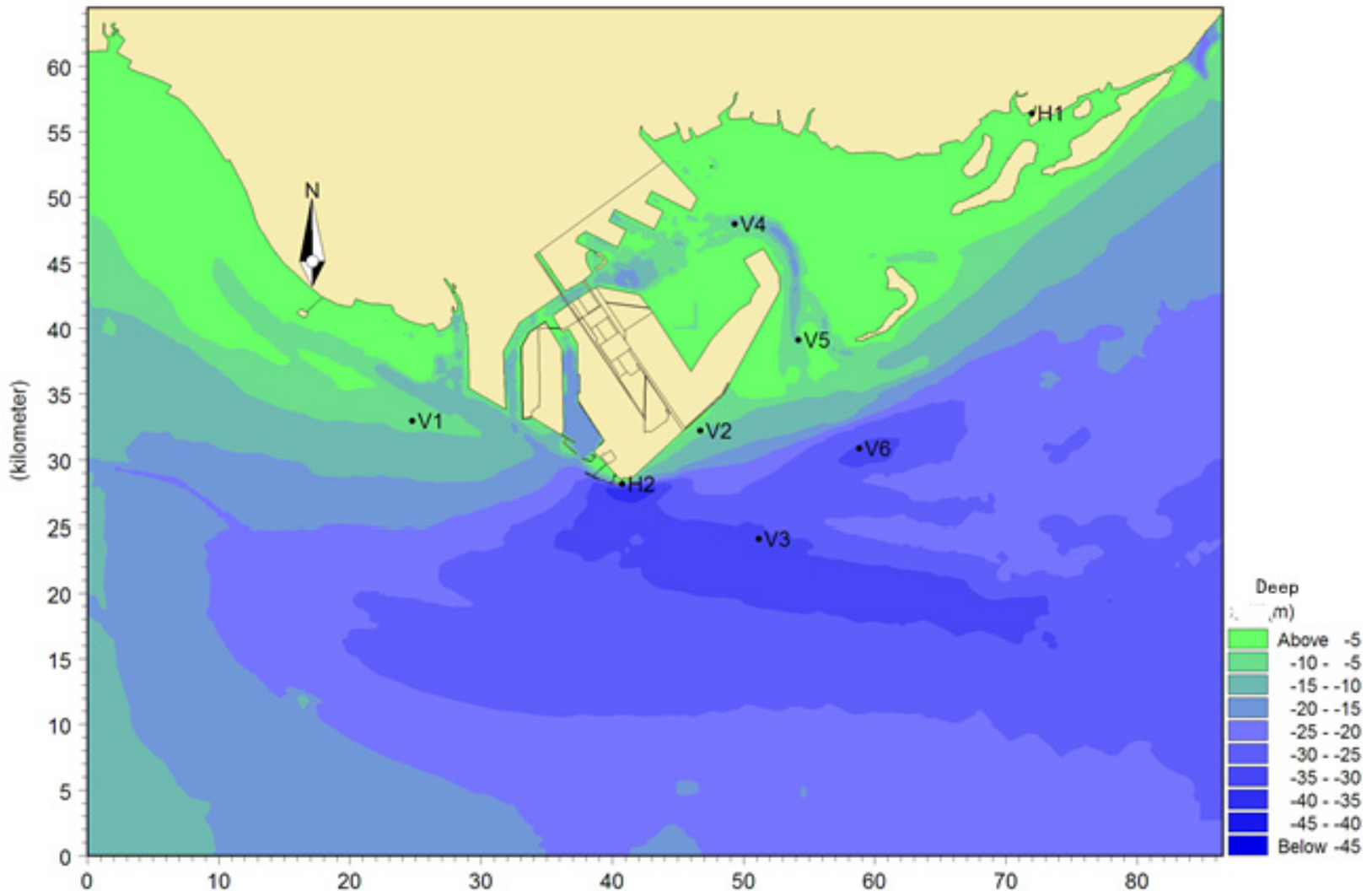

Fig 2 Water depth used for model

\subsection{FORECAST COMBINATION CONDITIONS}

According to the project's discharge situation, it is divided into different seasons in winter and summer, and the existing drainage situation in the surrounding area is further subdivided, and a combination plan of different discharge outlets is given. See Table 1 for details.

Table 1 Forecast portfolio

\begin{tabular}{|c|c|c|c|c|c|}
\hline Season & $\begin{array}{l}\text { Tide } \\
\text { Type }\end{array}$ & Scheme & $\begin{array}{l}\text { Intake and Drainage } \\
\text { Flow } \\
(\mathrm{m} 3 / \mathrm{s})\end{array}$ & $\begin{array}{l}\text { Drainage temperature rise } \\
\qquad\left({ }^{\circ} \mathrm{C}\right)\end{array}$ & Remarks \\
\hline \multirow[b]{2}{*}{ Summer } & \multirow[b]{2}{*}{ Full tide } & $\# 1$ & \multirow[b]{2}{*}{55.889} & \multirow[b]{2}{*}{10} & \multirow{2}{*}{$\begin{array}{c}\text { Superimpose China Resources } \\
\text { Summer } \\
\text { Displacement } 82.4 \mathrm{~m} 3 / \mathrm{s}, \\
\text { Temperature rise } 7.94^{\circ} \mathrm{C}\end{array}$} \\
\hline & & $\# 2$ & & & \\
\hline \multirow[b]{2}{*}{ Winter } & \multirow[b]{2}{*}{ Full tide } & $\# 1$ & \multirow[b]{2}{*}{37.5722} & \multirow[b]{2}{*}{14} & \multirow{2}{*}{$\begin{array}{c}\text { Superimpose China Resources } \\
\text { Winter } \\
\text { Displacement } 50.9 \mathrm{~m} 3 / \mathrm{s} \\
\text { Temperature rise } 10.25^{\circ} \mathrm{C}\end{array}$} \\
\hline & & $\# 2$ & & & \\
\hline
\end{tabular}

\section{RESULTS AND DISCUSSION}

\subsection{FORECAST RESULTS}

According to the prediction sequence of the temperature drainage plan, the two temperature discharge outlets to be selected are predicted for the full tide temperature discharge in summer and winter. After the prediction is stable, Fig3 shows the diffusion of warm water discharged through the discharge outlet of this project at high tide and low tide. Happening. It can be seen from the figure that both 1\# Warm Drain and 2\# Warm Drain are located at the intersection of Tidal channel and The third harbor, affected by tidal current, the temperature rise range of $4^{\circ} \mathrm{C}$ has little influence, and the temperature rise range of $1{ }^{\circ} \mathrm{C}$ spreads. The trend and scope are not much different. 


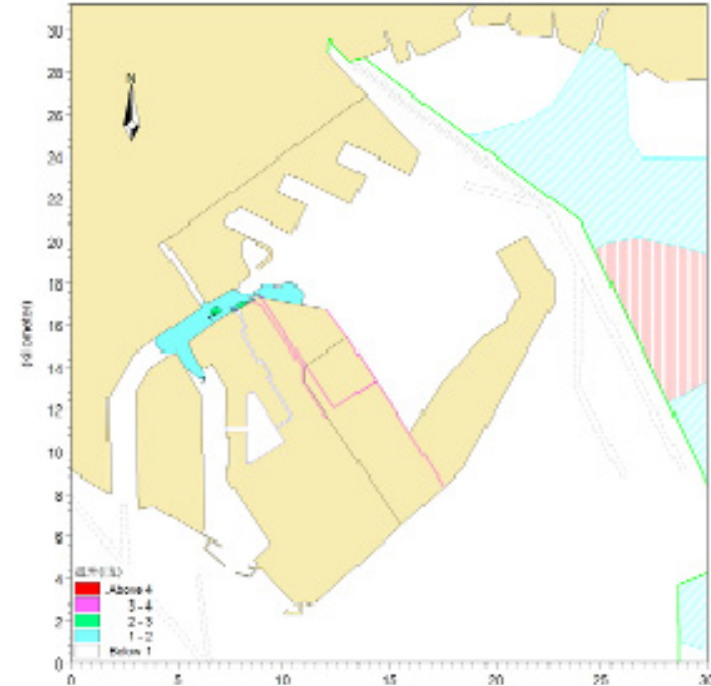

1\# Contour map of temperature rise at the time of rapid rise of temperature discharge outlet

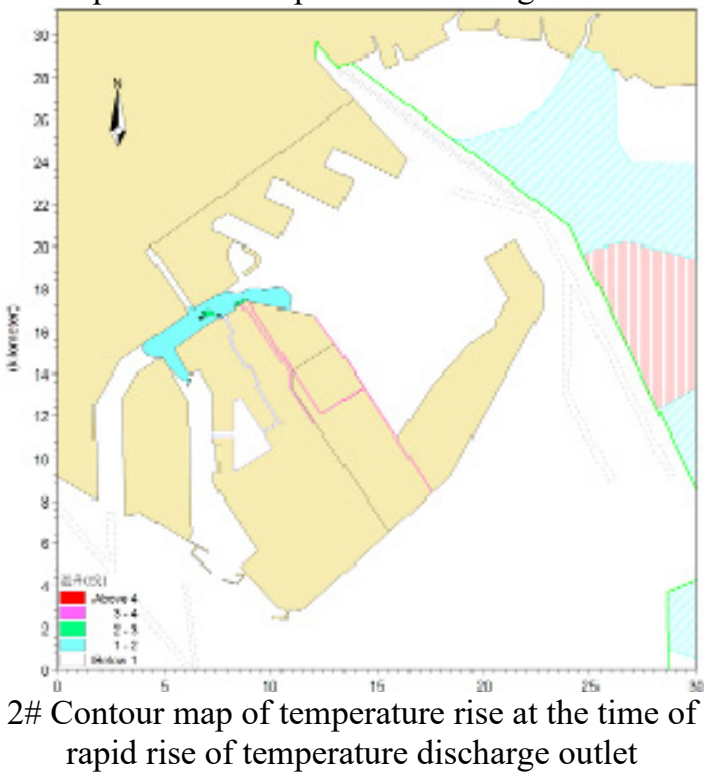

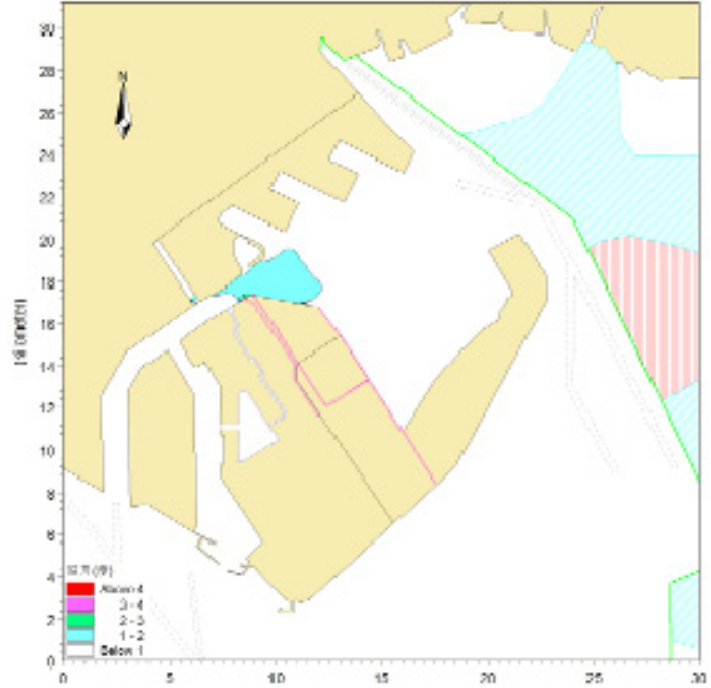

1\# Contour map of temperature rise at the moment of sudden drop of temperature discharge

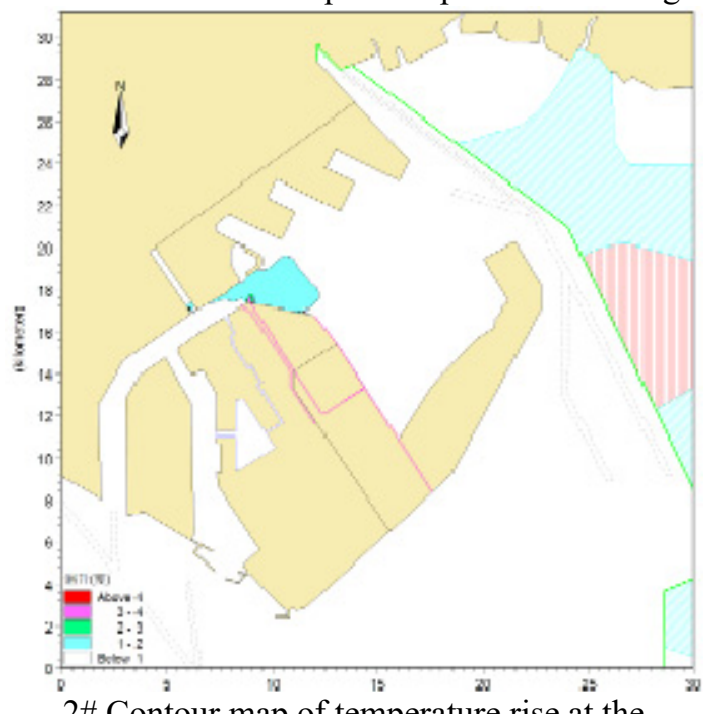

2\# Contour map of temperature rise at the moment of sudden drop of temperature discharge

Fig 3 Contour map of temperature rise at typical time in the sea area of the proposed temperature discharge outlet

It can be seen from the temperature rise contour map of the proposed temperature discharge outlet at a typical moment that the diffusion ranges of warm water at a certain instant after being discharged through the discharge outlet is relatively small, but the rapid rise and fall moments cannot fully represent the temperature rise. The influence of water masses at other times during the entire tide cycle. Therefore, in order to have a more comprehensive understanding of the scope of influence of warm water on the sea area after the discharge of warm water, this analysis has calculated the temperature rise package under the continuous full tide of high tide, medium tide and small tide. Network range, the predicted results are shown in Table 2 and Fig4 Fig7.

The proposed discharge outlets $1 \# \sim 2 \#$ are located on the shoreline between the warm discharge outlet of China Resources Power Plant and the liquefaction wharf. The discharge outlet drain is first discharged through the open channel and then discharged into the Tidal River (or San-gang Pond). This area is located in the harbor area of "Cao-feidian Port Shipping Area 2-6", so the sea water quality is not inferior to the four types of sea water quality standards (temperature rise does not exceed $4{ }^{\circ} \mathrm{C}$ ).

1\# Warm Drain is the location of the current temporary river course. This drain is located between the No. 1 Bridge and Cao Nan Railway Bridge. After the warm water is discharged through 1 \# Warm Drain, the $4^{\circ} \mathrm{C}$ temperature rise line at the water outlet of the summer tide will spread longitudinally. It is about $0.860 \mathrm{~km}$, the lateral diffusion distance is about $0.349 \mathrm{~km}$, and the maximum envelope area is $0.13 \mathrm{~km} 2$; the longitudinal diffusion distance of the $4{ }^{\circ} \mathrm{C}$ temperature rise line at the outlet of the full tide in winter is about $0.985 \mathrm{~km}$, and the lateral diffusion distance is about $0.290 \mathrm{~km}$. The area of the network is $0.16 \mathrm{~km} 2$; the influence trend and scope of the temperature rise during the full tide in summer and winter is not much different.

2\# Warm Drain is the location of the current Petrochemical Xihe River. After Petrochemical Xihe is reconstructed, it can meet the needs of warm drainage 
discharge. After predicting that the warm water is discharged through the 2\# Warm Drain, the $4^{\circ} \mathrm{C}$ temperature rise line at the full tide outlet in summer will spread longitudinally. It is about $.49 \mathrm{~km}$, the lateral diffusion distance is about $0.21 \mathrm{~km}$, and the maximum envelope area is $0.07 \mathrm{~km} 2$; the longitudinal diffusion distance of the $4^{\circ} \mathrm{C}$ temperature rise line at the outlet of the full tide in winter is about $0.805 \mathrm{~km}$, and the lateral diffusion distance is about $0.209 \mathrm{~km}$. The network area is $0.13 \mathrm{~km} 2$.

Table 2 The maximum temperature rise envelope area of the proposed discharge outlet at full tide ( $\mathrm{km} 2)$

\begin{tabular}{ccccccc}
\hline Season & Tide Type & Plan & $1{ }^{\circ} \mathrm{C} \sim 2^{\circ} \mathrm{C}$ & $2{ }^{\circ} \mathrm{C} \sim 3^{\circ} \mathrm{C}$ & $3{ }^{\circ} \mathrm{C} \sim 4^{\circ} \mathrm{C}$ & $>4^{\circ} \mathrm{C}$ \\
\hline \multirow{2}{*}{ Summer } & Full & $1 \#$ & 26.02 & 1.43 & 0.15 & $\mathbf{0 . 1 3}$ \\
& Full & $2 \#$ & 26.99 & 2.33 & 0.27 & $\mathbf{0 . 0 7}$ \\
\multirow{3}{*}{ Winter } & Full & $1 \#$ & 23.54 & 2.99 & 0.22 & $\mathbf{0 . 1 6}$ \\
& Full & $2 \#$ & 24.12 & 3.05 & 0.31 & $\mathbf{0 . 1 3}$
\end{tabular}

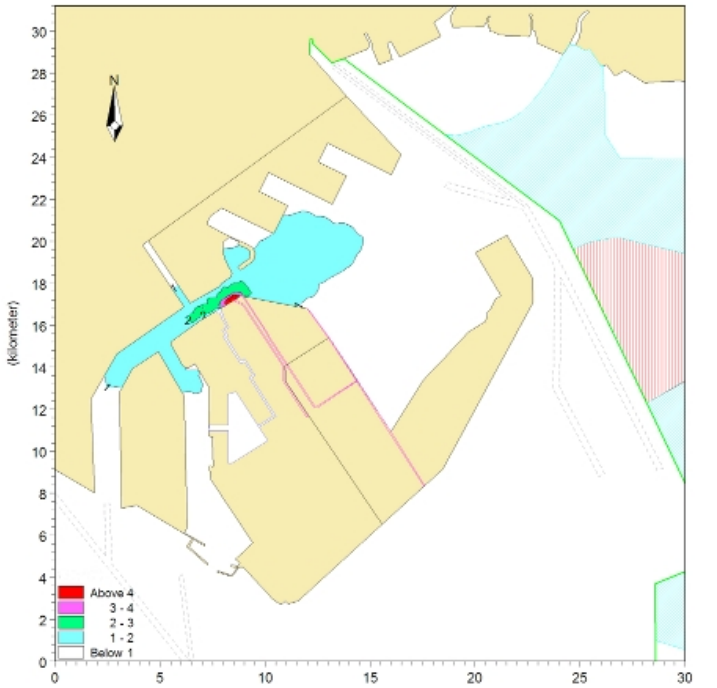

Fig 4 1\# Envelope diagram of the maximum temperature rise in the full tide at the location of the warm discharge outlet (Full tide in Summer)

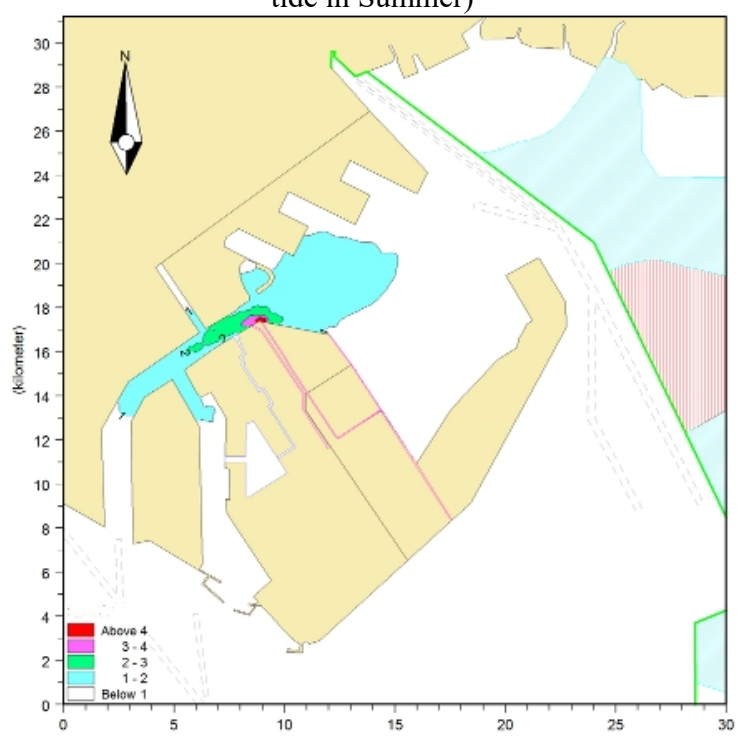

Fig5 2\# Envelop of the maximum temperature rise in the full tide at the location of the warm discharge outlet (large, medium, small tide in summer)

\subsection{DISCUSSION}

According to the analysis of this research, the coastline between the Wen Drain of China Resources Power Plant

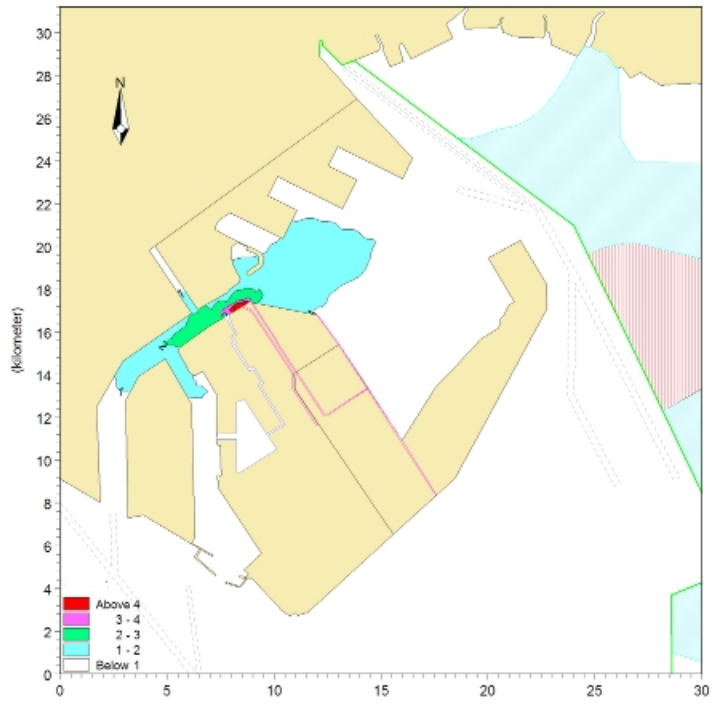

Fig 6 1\# Envelope diagram of the maximum temperature rise in the full tide at the location of the warm discharge outlet

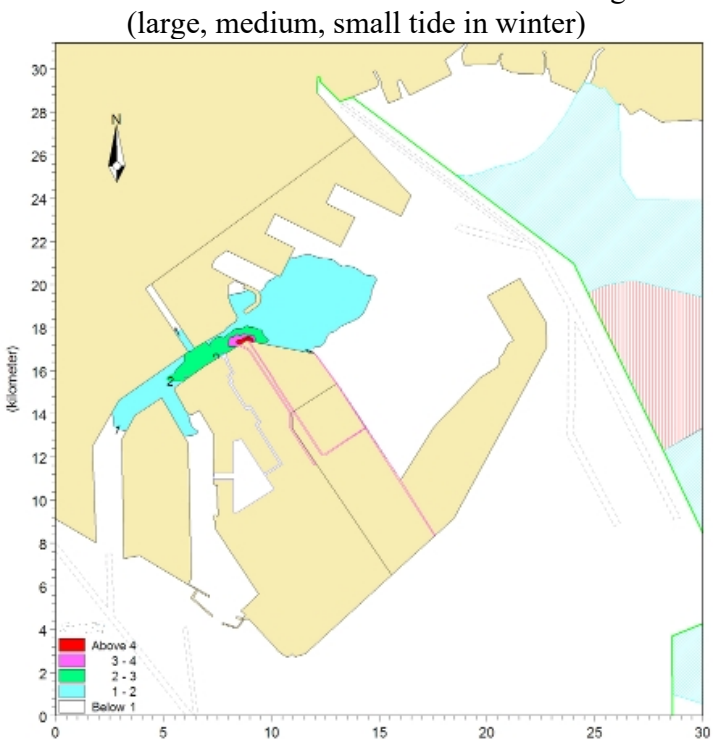

Fig7 2\# Envelop of the maximum temperature rise in the full tide at the location of the warm discharge outlet (winter large, medium and small tide)

on the north side of the Caofeidian Petrochemical Industrial Base and the West River of Petrochemical has selected 1\# and 2\# Wen Drain respectively for comparison and selection. 
By predicting the influence of the temperature rise of each proposed temperature exhaust outlet when it is discharged separately, it can be seen that:

(1) For the same planned temperature discharge outlet, the area affected by the $4^{\circ} \mathrm{C}$ temperature rise envelope of the summer full tide is equivalent to that of the winter full tide;

(2) The $1 \#$ and $2 \#$ warm discharge ports are located in the harbor area in the "Caofeidian Port Shipping Area 2-6", and the seawater quality is not inferior to the four types of seawater quality standards (temperature rise does not exceed $4^{\circ} \mathrm{C}$ ), 4 \#Wen Paikou is located in the sea area south of Caofeidian, and the sea water quality is not inferior to the second-class sea water quality standard (the temperature rise in summer does not exceed $1{ }^{\circ} \mathrm{C}$, and the other seasons do not exceed $2{ }^{\circ} \mathrm{C}$ );

(3) The warm water discharged from the $1 \#$ and $2 \#$ warm outlets is diluted and diffused by seawater, and the 2\# warm outlet has the smallest impact area. The maximum envelope area of the $4^{\circ} \mathrm{C}$ temperature rise in the winter at the full tide is $0.13 \mathrm{~km} 2$, followed by 1 \# For the warm outlet, the maximum envelope area of $4^{\circ} \mathrm{C}$ temperature rise is $0.16 \mathrm{~km} 2$. From the perspective of dilution and diffusion effect, the 2\# warm outlet has the strongest dilution and diffusion capacity for warm water under the current situation. Therefore, in this study, the No. 2 Wen Drainage outlet at Xihe Branch of Petrochemical Company was taken as the recommended discharge outlet.

\section{CONCLUSION}

(1) The Mike21 model is used this time to introduce the hydrological and topographic conditions of the area where a refining and chemical project is located, and a model is established to make a combined forecast of the different locations and discharge conditions of the temperature and discharge outlets of a refining and chemical project.

(2) By using the Mike21 model to predict various combination schemes, it can be known that the Mike21 model forecast results can clearly derive the influence range, which is helpful to judge the influence degree of different temperature drainage outlet setting schemes, so as to obtain the optimal scheme. Limited by the data conditions, the accuracy of the model is limited [5], but it can also provide a technical basis for the engineering design work, which can be applied in the plan selection stage.

\section{REFERENCES}

1. Cui Dan \& Jiang Zhibing. (2008). Numerical simulation of thermal drainage of power plants in coastal waters. Journal of Yangtze River Scientific Research Institute (02): 13-15.

2. Xu Ting. (2010). Mike21 hd calculation principle and application examples. Port Engineering Technology, 47(5): 1-5.
3. The State Council of China. (2012). Reply of the State Council on the Marine Functional Zoning of Hebei Province (2011-2020). Bulletin of the State Council of the People's Republic of China (30): 21.

4. Qi Jianwen, Kuang Cuiping, Jiang Mingtao, Deng Ling, Huo Rui \& Ma Zhen. (2014). The progress of Caofeidian port engineering and its threedimensional tidal field response characteristics. Hydrodynamic Research and Progress Series A (03): $346-354$.

5. Wang Qinggai, Dai Wennan, Zhao Xiaohong, Ding Feng, Li Shibei \& Zhao Yue. (2009). Numerical simulation study on thermal drainage of Laibin power plant expansion project based on Mike21FM. Environmental Science Research (03): 332-336. 